\title{
Introduction to series on technology-based HIV prevention and care interventions for youth
}

The iTech Center for Innovative Technology across the Prevention and Care Continuum is a collaborative center developed under the National Institute of Child Health and Human Development (NICHD)-funded, Adolescent Trials Network for HIV Interventions (ATN). iTech currently supports the implementation of ten (two completed, eight in progress) randomized controlled trials (RCTs) testing innovative technology-delivered interventions to address the HIV prevention and care continuum among youth in the United States (US) (1). Since 2018, nearly 1,700 youth have been enrolled across the US in either the formative intervention development phases or within the RCTs. iTech relies on a team science approach and the belief that substantial benefits can be gained by disseminating both the challenges and successes that have informed our collective experiences. The 11 articles included in this series of the journal explore an array of interconnected themes and provide a unique perspective on the development, implementation, evaluation and dissemination of technology-based interventions across the HIV prevention and care continuum.

Youth in the US, particularly Black and Latina/o/x sexual and gender minority (SGM) youth are disproportionately impacted by HIV (2). In their narrative review, Allan-Blitz et al., provide an overview of the HIV epidemic among youth using a developmental lens to highlight individual and structural drivers of HIV transmission and acquisition risk. Within this context, they present challenges and opportunities for new and innovative biobehavioral prevention and treatment interventions. As noted in their review, and in line with previous research, multiple intersecting forms of stigma and discrimination related to sex, gender, HIV, and race/ethnicity challenge HIV prevention and treatment service utilization, particularly among youth $(3,4)$. In their paper, Mulawa et al. review mobile health (mHealth) interventions that aim to reduce stigma as a way to improve HIV-related outcomes for youth. While the authors identified mHealth interventions that engage youth to reduce stigma, they contend that further efforts are needed, specifically calling for the development of theoreticallygrounded, multi-level interventions that not only measure HIV-related outcomes but also prioritize and measure changes in stigma and differentiate mechanisms through which various forms of stigma operate (5).

Sullivan et al. discusses how mobile applications (apps) and other mHealth tools fit into the current landscape of efficacious HIV prevention options for youth, highlighting both the unique strengths and limitations of what technology can do. Digital health interventions that are developmentally tailored to meet the needs of youth are highlighted as having the potential to increase uptake of prevention services including HIV and sexually transmitted infection (STI) testing. Biello et al. provide additional support for the utility of mobile apps to increase HIV and STI testing rates among youth, specifically among young gay, bisexual, and other men who have sex with men (YGBMSM). In their paper, the authors present the results from two pilot RCTs that were completed in iTech, each demonstrating that the distribution of HIV self-testing and STI self-collection kits was a feasible and acceptable strategy among YGBMSM.

Both the Sullivan and Biello papers emphasize the importance of conducting formative work to understand user characteristics and preferences when developing or adapting mHealth interventions for youth. This topic is explored further in the paper by Giovenco et al. which describes the results from a cross-cutting analysis of formative end-user research conducted with five samples of SGM youth living with or at-risk for HIV within iTech. Overall, feedback on preferred features and functions was consistent across the five interventions, and in line with prior research (6). Divergence, however, was seen in terms of content needs which varied based on the intervention's priority population, indicating that community-centered, cross-disciplinary, formative work should be conducted to ensure interventions are serving the diverse needs of SGM youth (7). This conclusion is reinforced in the paper by Skeen et al. which reviewed existing mobile technologies that were tailored exclusively for transgender and gender-expansive populations and incorporated gender-affirming features. Novel content and features that supported self-validation and promoted physical safety and emotional well-being were observed within platforms primarily originating outside the field of HIV prevention. These findings underscore the importance of harnessing genderaffirming cross-disciplinary innovations to advance the field.

Three studies focus on technology-driven methodologies that engage youth to support intervention development, implementation and evaluation. Gamarel et al., describe innovative approaches for online collection of formative qualitative 
data and discuss the relative advantages and disadvantages compared to face-to-face data collection methods. Virtual qualitative data collection allows for engagement of youth whose participation might be otherwise limited by structural barriers (e.g., lack of transportation, inconvenient timing) or a reluctance to participate due to stigma or discomfort with group settings. These methodologies may prove even more valuable given the impacts of the COVID-19 pandemic on research and health services delivery and requirements for physical distancing (8). Engaging youth to participate in mHealth studies also presents challenges. Lessons learned from recruitment of youth into four ongoing RCTs of mHealth HIV prevention interventions within $i T e c h$ are described in the paper by Zlotorzynska et al. In addition to providing metrics of success based on advertising and screening metrics, the authors also provide a framework for evaluation of data from future online recruitment efforts across platforms and geographic areas. Once enrolled, poor engagement can also limit the effectiveness of mHealth interventions for youth. In the paper authored by Hightow-Weidman et al., the author's discuss promising intervention strategies and features being employed within five ongoing iTech RCTs to enhance youth mHealth engagement, ensure its monitoring and reporting throughout the trial, and evaluate its impact on trial outcomes. A discussion of measurement approaches and metrics of success based on the engagement features embedded in the technology, key mechanisms of behavior change, and trial outcomes is provided.

The final two papers offer insights for both researchers new to the field wishing to create novel mHealth interventions, as well as for seasoned investigators wishing to scale-up established technology-based solutions. Siegler et al. provides a guide on how to maximize the opportunities mHealth tools and interventions afford, while avoiding salient pitfalls in mobile health app research and development. A critical issue is the mismatch between technology product development and research funding, both in terms of NIH budgets and timelines; the result of which is a manifested by a paucity of publicly available, "post-research" apps. Sharing and disseminating best-practices and leveraging collective experiences in multiple mHealth interventions, as is being done within $i$ Tech, may bring promising interventions to scale more quickly. Paul et al. discuss this topic further using as an example the app P3 (Prepared, Protected, em-Powered), an mHealth intervention to increase PrEP adherence among YGBMSM and young transgender women who have sex with men (9). The authors illustrate best practices and lessons learned for scale-up of mHealth interventions including the involvement of key stakeholders-particularly community members, potential implementers, and end-users-during initial planning, trials, analysis and dissemination. Capacity building (e.g., provision of staff training and technical assistance with deployment and evaluation) to support implementation of mHealth interventions by public health and community-based organizations is recommended.

The articles included in this series of the journal highlight the depth and breadth of youth-focused, mHealth intervention research that is being conducted both within iTech and in the broader research community. Ending the HIV epidemic among youth in the US will require an intentional, integrated and personalized approach that includes both behavioral and biomedical components and addresses the multi-level risk factors and barriers youth face. Technology-based platforms offer a potential way to deliver these integrated strategies in an engaging and impactful way and will assuredly play a key role in reducing HIV incidence and improving health outcomes among youth.

\section{Acknowledgments}

Funding: None.

\section{Footnote}

Provenance and Peer Review: This article was commissioned by the editorial office, mHealth for the series "Technology-based Interventions in HIV Prevention and Care Continuum among American Youth". The article did not undergo external peer review.

Conflicts of Interest: The author has completed the ICMJE uniform disclosure form (available at http://dx.doi.org/10.21037/ mHealth-2020-7). The series "Technology-based Interventions in HIV Prevention and Care Continuum among American Youth" was commissioned by the editorial office without any funding or sponsorship. LHW served as the unpaid Guest Editor of the series. Dr. Hightow-Weidman reports grants from NICHD, outside the submitted work. The author has no 
other conflicts of interest to declare.

Ethical Statement: The author is accountable for all aspects of the work in ensuring that questions related to the accuracy or integrity of any part of the work are appropriately investigated and resolved.

Open Access Statement: This is an Open Access article distributed in accordance with the Creative Commons AttributionNonCommercial-NoDerivs 4.0 International License (CC BY-NC-ND 4.0), which permits the non-commercial replication and distribution of the article with the strict proviso that no changes or edits are made and the original work is properly cited (including links to both the formal publication through the relevant DOI and the license). See: https://creativecommons.org/ licenses/by-nc-nd/4.0/.

\section{References}

1. Hightow-Weidman LB, Muessig K, Rosenberg E, et al. University of North Carolina/Emory Center for Innovative Technology (iTech) for Addressing the HIV Epidemic Among Adolescents and Young Adults in the United States: Protocol and Rationale for Center Development. JMIR Res Protoc 2018;7:e10365.

2. Centers for Disease Control and Prevention. HIV Among Youth. Last accessed: August 6, 2020. Available online: https://www. cdc.gov/hiv/group/age/youth/index.html

3. Arnold EA, Rebchook GM, Kegeles SM. 'Triply cursed': racism, homophobia and HIV-related stigma are barriers to regular HIV testing, treatment adherence and disclosure among young Black gay men. Cult Health Sex 2014;16:710-22.

4. Turan JM, Elafros MA, Logie CH, et al. Challenges and opportunities in examining and addressing intersectional stigma and health. BMC Med 2019;17:7.

5. Earnshaw VA, Smith LR, Chaudoir SR, et al. HIV stigma mechanisms and well-being among PLWH: a test of the HIV stigma framework. AIDS Behav 2013;17:1785-95.

6. Mulawa MI, LeGrand S, Hightow-Weidman LB. eHealth to Enhance Treatment Adherence Among Youth Living with HIV. Curr HIV/AIDS Rep 2018;15:336-49.

7. Gomez A, Loar R, Kramer AE, et al. Reaching and targeting more effectively: the application of market segmentation to improve HIV prevention programmes. J Int AIDS Soc 2019;22 Suppl 4:e25318.

8. Hightow-Weidman L, Muessig K, Claude K, et al. Maximizing Digital Interventions for Youth in the Midst of Covid-19: Lessons from the Adolescent Trials Network for HIV Interventions. AIDS Behav 2020;24:2239-43.

9. LeGrand S, Knudtson K, Benkeser D, et al. Testing the Efficacy of a Social Networking Gamification App to Improve PreExposure Prophylaxis Adherence (P3: Prepared, Protected, emPowered): Protocol for a Randomized Controlled Trial. JMIR Res Protoc 2018;7:e10448. 


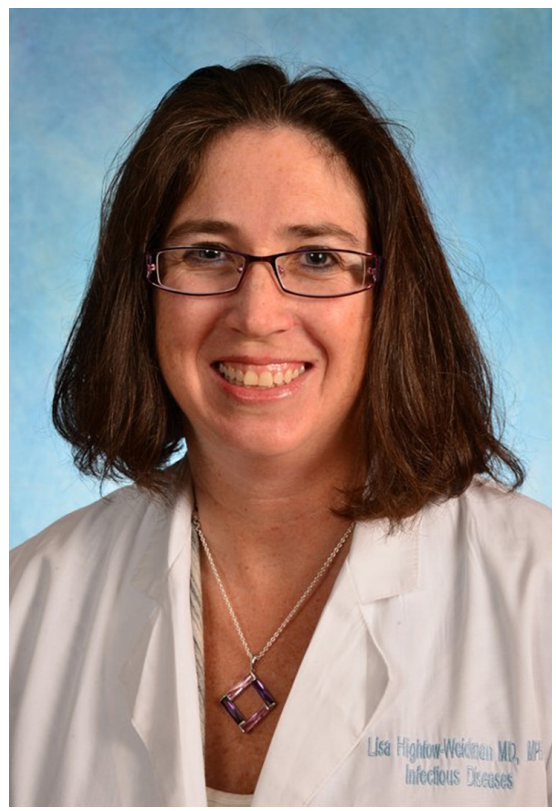

Lisa Hightow-Weidman

Lisa Hightow-Weidman, MD, MPH

Professor of Medicine and Health Behavior, Institute for Global Health and Infectious Diseases, University of North Carolina at Chapel Hill, Chapel Hill, NC, USA

(Email: Lisa_hightow@med.unc.edu)

Received: 10 August 2020; Accepted: 11 September 2020; Published: 20 July 2021. doi: $10.21037 / \mathrm{mHealth}-2020-7$ View this article at: http://dx.doi.org/10.21037/mHealth-2020-7

doi: $10.21037 / \mathrm{mHealth}-2020-7$

Cite this article as: Hightow-Weidman L. Introduction to series on technology-based HIV prevention and care interventions for youth. mHealth 2021;7:42. 